\title{
Rib 8
}

National Cancer Institute

\section{Source}

National Cancer Institute. Rib 8. NCI Thesaurus. Code C52760.

The eighth rib counting from the top of the rib cage down. 